\title{
Probing Crystalline Defects Using an EBSD-Based Virtual Dark-Field Method
}

Shiteng Zhao ${ }^{1,2}$, Ruopeng Zhang ${ }^{1,2}$, Thomas Pekin ${ }^{3}$ and Andrew M. Minor ${ }^{1,2 *}$

${ }^{1 .}$ National Center for Electron Microscopy, Molecular Foundry, Lawrence Berkeley National Laboratory, Berkeley, CA, USA.

2. Department of Materials Science \& Engineering, University of California, Berkeley, CA, USA.

3. Department of Physics, Humboldt-Universität zu Berlin, Berlin, Germany.

* Corresponding author: Andrew Minor; aminor@lbl.gov

Crystalline defects such as dislocations and grain boundaries are key features of interest when characterizing the mechanisms of plasticity in metals. To visualize these defects, Transmission Electron Microscopy (TEM) based techniques, with labor-intensive sample preparation procedures, are usually required. The result is typically a limited field of view and often an unrealistic defect morphology due to the lack of constraint in thin TEM samples. To address these problems, we propose to use an Electron Backscattered Scanning Diffraction (EBSD) -based technique combined with virtual aperture dark field imaging [1] to visualize defects in bulk materials. The proposed method has a potential to be a viable complement to TEM-based techniques.

Dislocations are the primary carrier of plasticity in metals. As important as they are, the visualization of dislocations is non-trivial and usually requires a careful choice of TEM imaging conditions, high-quality electron transparent foils and a limitation on the amount of material actually analyzed. SEM, on the other hand, provides a much larger field of view and can provide some information from the near subsurface of bulk materials. However, visualizing dislocations using SEM is very challenging, it requires the materials to be tilted in the way that the primary electron beam almost exactly fulfills the Bragg angle with one set of the lattice planes (similar to the two-beam condition in TEM), and so the electron backscattering is minimized. The electrons can then travel deep into the crystal through the channeling effect.

We propose to apply a new technique to rapidly and fully index crystalline defects based on Electron Channeling Contrast Imaging [2] and recently developed 4DSTEM analysis techniques [1]. The technique involves post-processing of the electron backscatter diffraction (EBSD) pattern (EBSP) and generation of dark-field type of images. The biggest advantage of this technique is that it can provide high throughput characterization by collecting the EBSP pattern in a large field of view. On the other hand, it does not require precise stage tilting to fulfill electron channeling criteria, but rather using virtual apertures one can selectively reconstruct the diffraction contrast. This method provides a fast, easy to implement, high throughput defect imaging alternative for materials science community.

We show here our preliminary results of a Ti-6wt.\%Al sample deformed by $4 \%$ of plastic strain. EBSD band contrast mapping (Fig. 1a) and inverse pole figure mapping (Fig. 1b) shows marginal contrast in the scanned area. It can only be vaguely seen in the band contrast image that there exist some line features, possibly dislocations generated by plastic deformation. However, by using the virtual dark field method, we can choose different "two beam" conditions and the contrast of the defects can be enhanced significantly, as shown in Fig. 1c-f. Note that the contrast of the image can be flipped by changing the sign of the excitation error, $\mathbf{s}$, which is consistent with diffraction contrast in conventional TEM dark field imaging. 
It is our ultimate goal to characterize dislocations using this EBSD-based virtual dark field technique in a bulk SEM sample and quantitatively measure the strain fields [3] and local lattice change around dislocations. This SEM-based method will provide a viable complement to TEM-based techniques such as 4D-STEM and precession electron diffraction. Due to the relatively larger chamber in an SEM, multiple diagnostics such as secondary electron, backscattered electron, Energy Dispersive Spectroscopy (EDS) can function at the same time, leading to a high throughput dataset of a bulk sample including its morphological, structural, orientational and chemical information. This dataset, plus the direct visualization of crystalline defects using virtual apertures, will help to enhance our comprehension of the underlying mechanisms of plastic deformation.

\section{References:}

[1] C Gammer et al., Ultramicroscopy 155 (2015).

[2] N Brodusch, H Demers and R Gauvin., Ultramicroscopy 148 (2015).

[3] VB Ozdol et al., Applied Physics Letters 106 (2015).
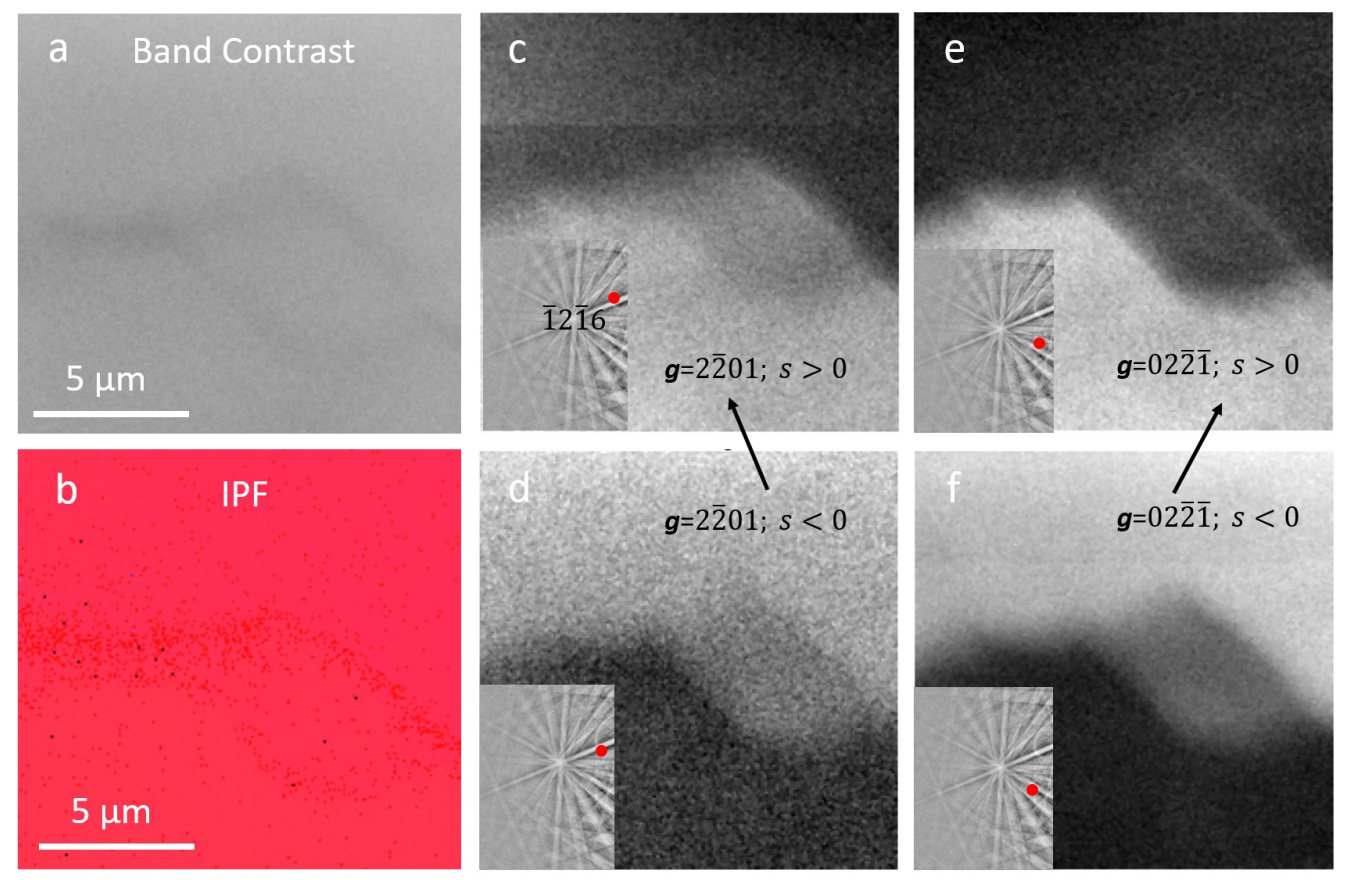

Figure 1. Conventional EBSD band contrast mapping (a); inverse pole figure (b); and post-processed virtual dark field images using different two beam condition and excitation error (c-f). 